patients with primary APS, 54 patients with positive aPL serology not meeting clinical criteria for APS and 326 healthy controls adjusted by the month of vitamin $\mathrm{D}$ analysis. We considered $30 \mathrm{ng} / \mathrm{ml}$ and $10 \mathrm{ng} / \mathrm{ml}$ as the thresholds for vitamin $D$ insufficiency and deficiency, respectively.

Results: Median levels of vitamin D were similar in the three groups: 21 (range 5-69) in primary APS, 25 (4-50) in the aPL-positive group, and 21 (4-105) in controls. Overall, $53.9 \%$ of measurements were performed during the sunny season (April to September). Ten percent of patients with primary APS were males, versus $16 \%$ in the aPL serology group and $26 \%$ among healthy controls $(p=0.007)$. Mean age was $46 \pm 15$ in primary APS, $49 \pm 17$ in the aPL-positive group and $53 \pm 10$ in the control group $(p<0.001)$. Regarding vitamin $D$ insufficiency, $82 \%$ of APS patients had levels of vitamin D $(<30 \mathrm{ng} / \mathrm{ml})$ versus $70 \%$ and $72 \%$ of patients with aPL serology and controls, respectively $(p=0.168)$. When analyzing the prevalence of vitamin D deficiency $(<10 \mathrm{ng} / \mathrm{ml})$, we found significant differences across the groups: $16.2 \%$ in patients with primary APS, $11.1 \%$ in patients with positive serology and only $4.9 \%$ in healthy controls $(p=0.002)$. There was no significant association between insufficient levels of vitamin $D$ and the presence of thrombotic or obstetric events. Nevertheless, we found a trend for the presence of more thrombotic events in patients with vitamin $D$ deficiency $(p=0.097)$. Regarding the immunological profile, we found no association between vitamin $\mathrm{D}$ and either the number of positive antibodies or their serological evolution. However, we found an association between insufficient levels of vitamin $\mathrm{D}$ and the presence of lupus anticoagulant $(54.7 \%$ vs $18.2 \%, p=0.047)$

Conclusions: More than $80 \%$ of patients with primary APS have insufficient levels of vitamin $D$ and $16 \%$ of them have very low levels of vitamin $D$.

Primary APS patients show a higher frequency of vitamin D deficiency than healthy controls.

Patients with vitamin $D$ insufficiency have more commonly positivity for lupus anticoagulant.

Disclosure of Interest: None declared

DOI: 10.1136/annrheumdis-2017-eular.5129

\section{AB0512 EPIDEMIOLOGICAL, CLINICAL AND IMMUNOLOGICAL CHARACTERISTICS OF ANTIPHOSPHOLIPID SYNDROME: STUDY OF 170 PATIENTS}

L. Riancho-Zarrabeitia, S. García-Canale, M. Cubería, G. Daroca, M. López-Hoyos, P. Muñoz, M. Agudo, V. Martínez-Taboada. Hospital Universitario Marqués de Valdecilla, Santander, Spain

Background: Antiphospholipid syndrome (APS) is an autoimmune disease defined by the presence of antiphospholipid antibodies ( $\mathrm{aPL}$ ) and thrombosis and/or pregnancy morbidity. Although thrombotic and obstetric APS are considered the same disorder, there are pathogenetic and clinical differences between them. Objectives: To describe the epidemiological, clinical and immunological characteristics of a cohort of APS patients from a defined population and to study the differences between thrombotic, obstetric and mixed APS.

Methods: Retrospective study including patients attending the rheumatology and the obstetric clinics of a tertiary facility in Northern Spain. All patients met APS classification criteria.

Results: We included 84 patients with thrombotic APS, 76 with obstetric APS and 10 with mixed APS. Main demographical characteristics are showed in table. There were differences in the age of discovery a positive serology ( $46 \pm 15 \mathrm{yr}$ in thrombotic APS, $36 \pm 8 \mathrm{yr}$ in obstetric, and $36 \pm 14$ in mixed APS). Moreover, the prevalence of systemic lupus erythematosus (SLE) was higher in patients with thrombotic and mixed APS ( $26 \%$ and $30 \%$ vs $5 \%$ in obstetric APS, $p=0.001)$. Anticardiolipin antibodies were, overall, the most frequently positive. Lupus anticoagulant was significantly more common in patients with thrombotic and mixed APS $(70 \%$ and $71 \%$ vs $30 \%$ in obstetric APS, $p=0.002$ ). We found no differences in the load

\begin{tabular}{lccccc}
\hline & Total & Thrombotic APS & Obstetric APS & Mixed APS & P \\
& 170 & 84 & 76 & 10 & \\
\hline Age (yr), mean \pm SD & $41,2 \pm 13,7$ & $46.5 \pm 15.5$ & $35.9 \pm 8.1$ & $36.5 \pm 14.4$ & $<0.001$ \\
SLE, $\mathrm{n}(\%)$ & $29(17)$ & $22(26)$ & $4(5)$ & $3(30)$ & 0.001 \\
Load of antibodies & & & & 0.201 & \\
-1 & $63(37)$ & $24(29)$ & $34(45)$ & $5(50)$ & \\
-2 & $68(40)$ & $37(44)$ & $28(37)$ & $3(30)$ & \\
-3 & $38(22)$ & $23(27)$ & $13(17)$ & $2(20)$ & \\
aCL & $130(77)$ & $67(80)$ & $54(72)$ & $9(90)$ & 0.306 \\
aB2Gp1 & $108(64)$ & $56(67)$ & $49(65)$ & $3(30)$ & 0.070 \\
LA & $53(58)$ & $40(70)$ & $8(30)$ & $5(71)$ & 0.002 \\
Family history of & & & & & \\
$\quad$ thrombosis, $\mathrm{n}(\%)$ & $21(19)$ & $14(29)$ & $4(7)$ & $3(50)$ & 0.011 \\
Traditional CV risk factors, $\mathrm{n}(\%)$ & $69(41)$ & $37(44)$ & $28(37)$ & $4(40)$ & 0.650 \\
$\quad$ Tobacco use & $55(32)$ & $41(49)$ & $10(13)$ & $4(40)$ & $<0.001$ \\
$\quad$ Hypertension & $48(28)$ & $39(46)$ & $5(7)$ & $4(40)$ & $<0.001$ \\
Dyslipidemia & $8(5)$ & $4(5)$ & $4(5)$ & 0 & 0.761 \\
$\quad$ Diabetes mellitas & & & & & \\
Previous treatment, $\mathrm{n}(\%)$ & $88(52)$ & $28(33)$ & $54(72)$ & $6(60)$ & $<0.001$ \\
$\quad$ Heparin & $71(42)$ & $62(74)$ & $3(4)$ & $6(60)$ & $<0.001$ \\
$\quad$ Oral anticoagulants & $75(86)$ & $63(76)$ & $74(97)$ & $8(80)$ & $<0.001$ \\
Antiplatelet therapy & $145)$ & $5(6)$ & 0 & 0 & 0.072 \\
Corticoids & $5(3)$ & $31(37)$ & $13(17)$ & $3(30)$ & 0.020 \\
Antimalarials & $47(28)$ & $4(5)$ & $1(1)$ & 0 & 0.371 \\
$\quad$ Immunosuppressants & $5(3)$ & & & & \\
\hline & & & &
\end{tabular}

of antibodies between the three groups. Regarding traditional cardiovascular risk factors (CVRF), tobacco use was the most common, followed by hypertension and dyslipidemia. The last two factors were more frequent in patients with thrombotic and mixed APS than in those with obstetric APS $(p<0.001)$. As expected, treatment with heparin was more frequent in obstetric and mixed APS, while oral anticoagulants were more frequently used in thrombotic APS. Antimalarial drugs were less frequently used in obstetric APS ( $17 \%$ vs $37 \%$ and $30 \%, p=0.020)$, probably due to a lower prevalence of lupus in this group.

Conclusions: In our cohort, patients with thrombotic or mixed APS have a higher frequency of SLE than patients with obstetric APS. Positivity for lupus anticoagulant is more common in patients with thrombotic or mixed APS. Regarding traditional CVRF, hypertension and dislypidemia are more common in patients with thrombotic or mixed APS.

Disclosure of Interest: None declared

DOI: 10.1136/annrheumdis-2017-eular.4338

\section{AB0513 FATIGUE IN CHINESE PATIENTS WITH PRIMARY SJÖGREN'S SYNDROME: A CROSS SECTIONAL STUDY}

L. Li ${ }^{1}$, Y. Cui ${ }^{1}$, S. Chen ${ }^{1}$, Q. Zhao ${ }^{1}$, L. Li ${ }^{2}$, Z. Gu ${ }^{1} .{ }^{1}$ Department of Rheumatology, Affiliated Hospital of Nantong University; ${ }^{2}$ School of Nursing of Nantong University, Nantong, China

Background: Primary Sjögren's syndrome (pSS) is the second most common systemic autoimmune disease, with a female-male ratio of $9: 1$, and characterized by sicca symptoms of the eyes and mouth, including joint pains and multi-system involvement. pSS affects patients' health-related quality of life (QoL), mental status and relationships with family. In pSS patients, symptoms such as fatigue, depression, arthralgia, fibromyalgia and general loss of well-being are commonly reported. Among them, fatigue is the most common problem that includes physical and mental fatigue, it can be as disabling as pain, which is difficult to manage and has a notable impact on QoL. Fatigue is a tiredness which may be mental, physical, or both, and that results in an inability to function at normal performance levels. However, the underlying pathophysiological mechanisms of fatigue remain unclear. A number of studies have reported the association of fatigue with Primary Sjögren's syndrome (pSS), whereas, because of the small sample size of pSS patients, we still lack large sample studies to find the relationship between pSS and fatigue.

Objectives: To investigate the relationship of fatigue severity to other clinical features in primary Sjögren's syndrome (pSS) and to identify factors contributing to the physical and mental aspects of fatigue in Chinese patients.

Methods: Sixty-seven consecutive patients with PSS according to the AmericanEuropean Consensus group (AEGG) criteria were included. Demographic, clinical and biological characteristics for all patients were collected. The Fatigue Severity Scale (FSS), Profile of Fatigue (ProF), Visual analogue scale, Hospital Anxiety and Depression Scale (HADS), OHIP-14 Scale, MDADI Scale and PSQI Scale were adopted to assess fatigue, depression, anxiety, xerostomia, xerophthalmia and sleep disturbances. Associations with fatigue were compared using multivariate regression.

Results: $94 \%$ of our patients were women. The mean age of patients was $51.13 \pm 13.23$ years, and the mean disease duration was $4.12 \pm 4.49$ years. The mean oral dryness was $51 \pm 17.82$, and the mean ocular dryness was $33.56 \pm 26.3$. Abnormal fatigue, defined as an FSS score $>0 \mathrm{or}=4$, was present in $64 \%$ of the patients. Dry symptoms, low educational level, Pain and depression had a negative impact on fatigue scores. The regression models explained that Pain and depression were the strongest predictors of fatigue according to the FSS.

Conclusions: Fatigue is a tiredness which may be mental, physical, or both, and that results in an inability to function at normal performance levels. However, the underlying pathophysiological mechanisms of fatigue remain unclear. From our study, we found that psychosocial variables are determinants of fatigue, and fatigue is associated with depression, but depression is not the primary cause of fatigue in primary SS. Therefore, the investigation of the pathophysiologic correlates of physical and mental aspects of fatigue is needed to guide the development of more effective interventions.

Acknowledgements: This study was supported by National Natural Science Foundation of China (81401124); the Collaborative Innovation Program of Affiliated Hospital of Nantong University; College graduate research and innovation of Jiangsu Province (KYZZ16-0358, KYZZ15-0353).

Disclosure of Interest: None declared

DOI: 10.1136/annrheumdis-2017-eular.3076

\section{AB0514 THE RELATIONSHIP BETWEEN SERUM LEVEL OF C-TERMINAL TELOPEPTIDE OF TYPE I COLLAGEN WITH COURSE OF SYSTEMIC LUPUS ERYTHEMATOSUS AND STRUCTURAL AND FUNCTIONAL STATE OF BONE TISSUE}

S. Shevchuk, L. Denyschych, L. Marynych. National Pirogov Memorial Medical University, Vinnytsya, Ukraine, Vinnytsya, Ukraine

Background: It is well known, that the incidence of osteoporosis in patients with systemic lupus erythematosus (SLE) is higher compared to the population level. Its severity in patients with SLE is associated with a number of factors: female gender, disease activity, damage index, glucocorticoid therapy etc. One of metabolic factors indicating the reducing bone mineral density (BMD) is the level 